\title{
Significance of the Pars Interarticularis in the Cortical Bone Trajectory Screw Technique: An In Vivo Insertional Torque Study
}

\author{
Koshi Ninomiya, Koichi Iwatsuki, Yu-Ichiro Ohnishi, Toshika Ohkawa, Toshiki Yoshimine \\ Department of Neurosurgery, Osaka University Graduate School of Medicine, Suita, Japan
}

\begin{abstract}
Study Design: Retrospective study.
Purpose: Cortical bone trajectory (CBT), a more medial-to-lateral and shorter path than the traditional one for spinal fusion, is thought to be effective for severely degenerated vertebrae because screws are primarily stabilized at the posterior elements. We evaluated the efficacy of this approach through in vivo insertional torque measurement.

Overview of Literature: There has been only one prior in vivo study on CBT insertional torque.

Methods: Between January 2013 and April 2014, a total of 22 patients underwent posterior lumbar fusion using the CBT technique. The maximum insertional torque, which covers the radial strength needed for insertion, was measured for 113 screws, 8 of which were inserted for $L 5$ spondylolysis. The insertional torque for cases with $(n=8)$ and without $(n=31)$ spondylolysis of $L 5$ were compared using one-way analysis of variance (ANOVA). To evaluate vertebral degeneration, we classified 53 vertebrae without spondylolysis by lumbar radiography using semiquantitative methods; the insertional torque for the 105 screws used was compared on the basis of this classification. Additionally, differences in insertional torque among cases grouped by age, sex, and lumbar level were evaluated for these 105 screws using ANOVA and the Tukey test.

Results: The mean insertional torque was significantly lower for patients with spondylolysis than for those without spondylolysis $(4.25$ vs. 8.24 in-lb). There were no statistical differences in insertional torque according to vertebral grading or level. The only significant difference in insertional torque between age and sex groups was in men $<75$ years and women $\geq 75$ years ( $10 \mathrm{vs.} 5.5 \mathrm{in}-\mathrm{lb}$ ). Conclusions: Although CBT should be used with great caution in patient with lysis who are $\geq 75$ years, it is well suited for dealing with severely degenerated vertebrae because the pars interarticularis plays a very important role in the implementation of this technique.
\end{abstract}

Keywords: Cortical bone trajectory; Insertional torque; Spondylolysis; Pars interarticularis; Vertebral degeneration

\section{Introduction}

The cortical bone trajectory (CBT) technique, used with a pedicle screw, was first described by Santoni et al. [1] in 2009. The trajectory starts in the lateral part of the pars interarticularis and follows a caudocephalad and lateral path through the pedicle. We began using this method in our institution (Department of Neurosurgery, Osaka University Graduate School of Medicine) in 2012 for cases requiring thoracolumbar fusion. Compared with traditional

Received Jun 15, 2015; Revised Jun 30, 2015; Accepted Jun 30, 2015

Corresponding author: Koshi Ninomiya

Neurosurgery Suita, Osaka University Graduate School of Medicine, Osaka University,

Neurosurgery, Yamadaoka 2-2, Osaka 565-0871, Japan

Tel: +81-6-6879-3652, Fax: +81-6-6879-3659, E-mail: k-ninomiya@nsurg.med.osaka-u.ac.jp 
methods of inserting a pedicle screw, CBT is thought to be more effective for initial fixation by maximizing contact between the screw and cortical bone. In addition, it may also be useful in dealing with severely degenerated vertebrae in patients with osteoporosis because the screws are primarily stabilized at the posterior elements and are not deeply inserted into the vertebral bodies.

However, there have been few evaluations with respect to stabilization of the CBT screw in cadaver or in vivo studies. Therefore, it has yet to be determined whether $\mathrm{CBT}$ is truly indicated for patients with osteoporosis. To the best of our knowledge, there has been only one prior in vivo study on CBT insertional torque [2]. This study was designed to investigate the efficacy of the CBT technique and appropriate structures for its use through the measurement of in vivo insertional torque.

\section{Materials and Methods}

This study was approved by our Institutional Ethics Committee (approval number 14168).

Between January 2013 and April 2014, a total of 22 consecutive patients, including 9 men (mean age, $61.0 \pm 15.7$ years; range, 37-80 years) and 13 women (mean age, $62.3 \pm 14.6$ years; range, $32-80$ years) underwent posterior interbody or posterior lumbar fusion at our institution using the CBT technique for the treatment of lumbar degenerative spondylolisthesis, lumbar canal stenosis, or lumbar spondylolysis. In total, 114 screws were inserted into the lumbar spine. Among these, eight were inserted for cases with L5 spondylolysis.

CBT was performed under lateral fluoroscopy. We used the isthmus of the lamina as an anatomical landmark for entry [3]. Screws were placed $3 \mathrm{~mm}$ inside the isthmus and inserted in a cephalad and lateral direction. In cases with spondylolysis, the entry point for the screws was the rostral point of the defect in the pars interarticularis; the trajectory followed was the same as that in the CBT technique. In both cases, similar tapping was done. The screw diameter was $4.5 \mathrm{~mm}$ (Zodiac polyaxial screw, Alphatec Spine, Tokyo, Japan), the screw lengths were 25, 30, and 35 $\mathrm{mm}$, and the pitch was $4 \mathrm{~mm}$. The maximum insertional torque of the last two screw rotations was measured using an Inline dial indicator 584100 (Holmed Corporation, Franklin, USA) (Fig. 1). Its scale was modified by Alphatec Spine to measure $0-55 \mathrm{in}-\mathrm{lb}$ in increments of $1 \mathrm{in}-\mathrm{lb}$.

The insertional torque of the 114 screws used in our study was also measured. One of them was incorrectly placed medially by more than half of its diameter; therefore, it was excluded from our study. The insertional torques in cases with $(\mathrm{n}=8)$ and without $(\mathrm{n}=31)$ spondylolysis of L5 were compared using one-way analysis of variance (ANOVA).

To evaluate vertebral degeneration, 53 vertebrae (excluding cases with spondylolysis) were classified based on lumbar radiographs using a semiquantitative method [4]. This is an quick and routine assessment of vertebral fractures [5] and correlates moderately well with quantitative morphometry [6]. Vertebrae were graded on visual inspection and without direct vertebral measurement as follows: (1) Normal (grade 0), (2) Mildly deformed (grade 1 , approximately $20 \%-25 \%$ reduction in anterior, middle, and/or posterior height and a reduction in area of $10 \%-$ 20\%), (3) Moderately deformed (grade 2, approximately $25 \%-40 \%$ reduction in any height and a reduction in area of $20 \%-40 \%$ ), (4) Severely deformed (grade 3 , more than an approximate $40 \%$ reduction in any height and area).

There were 14 vertebrae of grade 0,23 vertebrae of grade 1 , and 16 vertebrae of grade 2 ; there were no grade 3 vertebrae in this study. The insertional torque of the 105 screws used for these 53 vertebrae was statistically compared using one-way ANOVA with the Tukey test.

To assess the relation among sex, age, and insertional torque, the 105 screws were classified into four groups based on patient age and sex: men $<75$ years $(n=24)$, women $<75$ years $(n=56)$, men $\geq 75$ years $(n=10)$, and women $\geq 75$ years $(n=15)$. According to a previous study [7] using a quantitative method, the prevalence of vertebral fracture in women rapidly increases in their 70s, reflecting lower bone density. To evaluate the same kind of sex- and age-related tendency in posterior elements, we chose 75 years, termed as late elderly in Japan, as the division criterion.

In addition, insertional torque and lumbar level (L1,

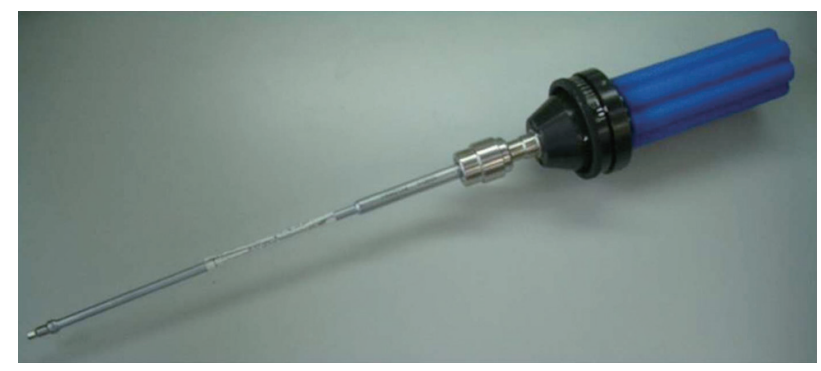

Fig. 1. Photograph of the torque measurement device. 
$\mathrm{n}=2 ; \mathrm{L} 2, \mathrm{n}=10 ; \mathrm{L} 3, \mathrm{n}=22 ; \mathrm{L} 4, \mathrm{n}=40$; and L5, $\mathrm{n}=31$ ) were evaluated for these 105 screws statistically using one-way ANOVA with the Tukey test.

\section{Statistical analysis}

Statistical analyses were performed using one-way ANOVA with or without the Tukey test. Statistical significance was defined as $p<0.05$. We used JMP pro ver. 11 (SAS Institute
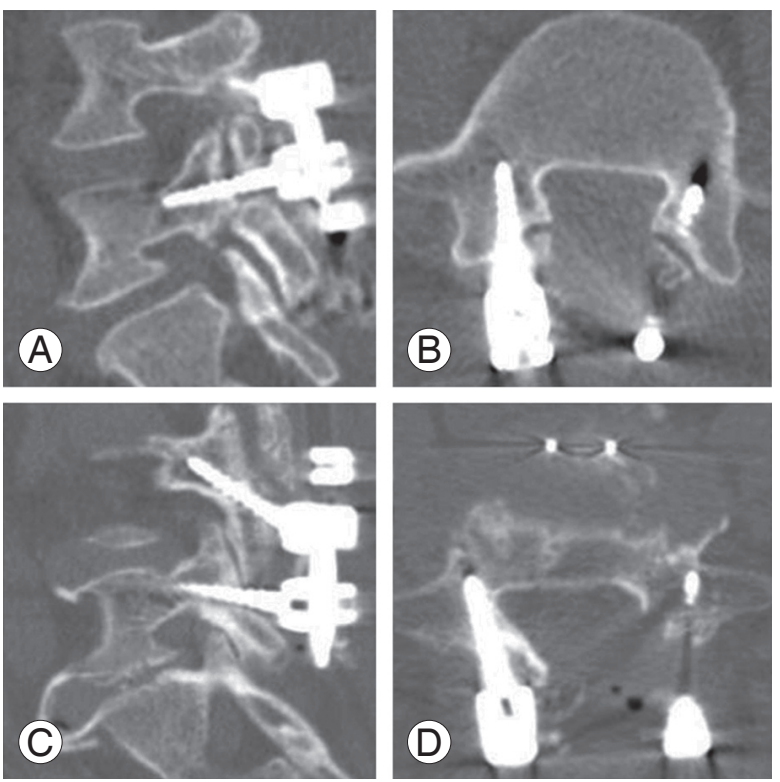

Fig. 2. Postoperative computed tomography demonstrating typical screw insertion in L5: sagittal view (A) and axial view (B) of L5 screws for a case with spondylolysis. Sagittal view (C) and axial view (D) for a case without spondylolysis.
Japan, Tokyo, Japan) for statistical analysis.

\section{Results}

Typical cases with and without spondylolysis of L5 are shown in Fig. 2.

The mean maximum insertional torque in L5 for cases with spondylolysis was significantly lower than that for cases without spondylolysis $(4.25 \pm 1.74$ vs. $8.24 \pm 0.89$ inlb, respectively; $p=0.040$ ) (Fig. 3).

Among the four groups classified by sex and age, there was a significant difference only between insertional torque for men $<75$ years and women $\geq 75$ years $(10 \pm 1.03$ vs. $5.5 \pm 1.31$ in-lb, respectively; $p=0.038$ ) (Fig. 4 ).

The mean insertional torque by grade of vertebral de-

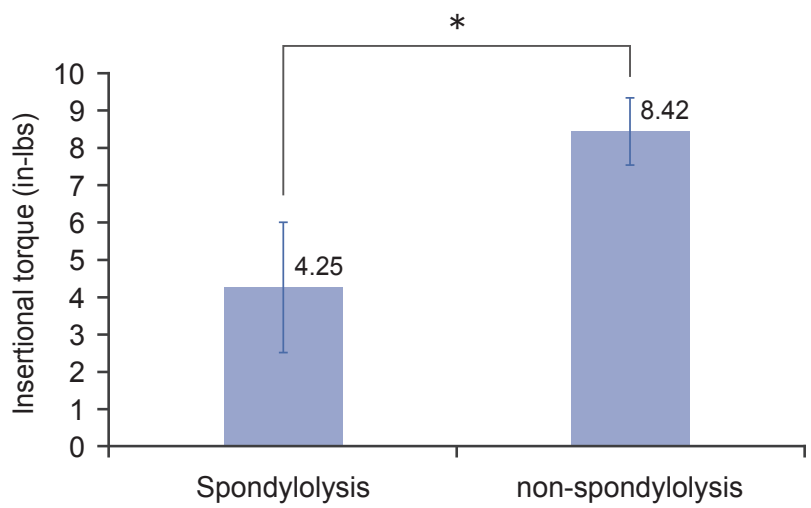

Fig. 3. The mean \pm SEM for maximum insertional torque in cases with and without spondylolysis in L5 ( $p=0.040)$. SEM, standard error of mean.

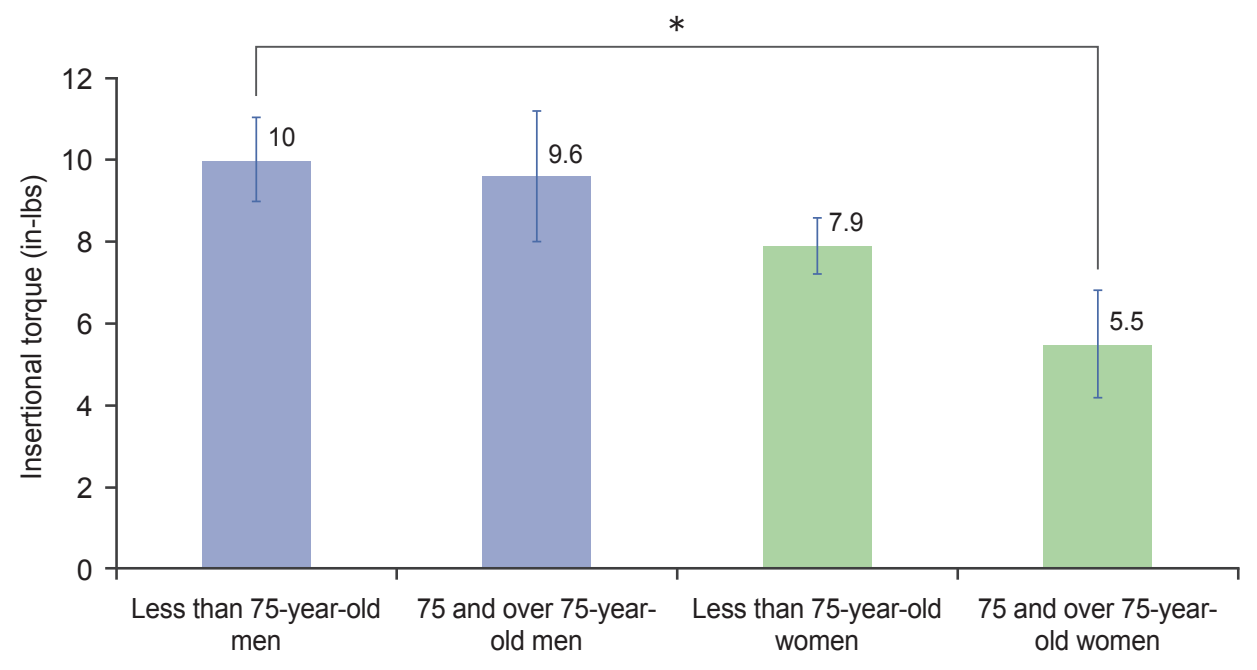

Fig. 4. The mean $\pm S E M$ for maximum insertional torque by age and sex group. Significantly greater torque was used by men $<75$ years than women $\geq 75$ years $(p=0.038)$. SEM, standard error of mean. 
generation is shown in Fig. 5. The mean insertional torque for grade 0,1 , and 2 vertebrae was $8.46 \pm 0.83,8.58 \pm 0.73$, and $7.31 \pm 0.76$ in-lb, respectively. There was no statistical difference among them.

The mean insertional torque according to lumbar vertebral level is shown in Fig. 6. There were no statistical differences among them.

None of the patients in our study experienced surgical complications.

\section{Discussion}

Santoni et al. [1] designed the CBT technique to attach surgical screws to high-density bones in the posterior aspect of the spinal column, and they evaluated its efficacy in their human cadaver study. They demonstrated a 30\% increase in uniaxial yield pullout load and equivalency in mixed loading for the CBT screw compared with that for

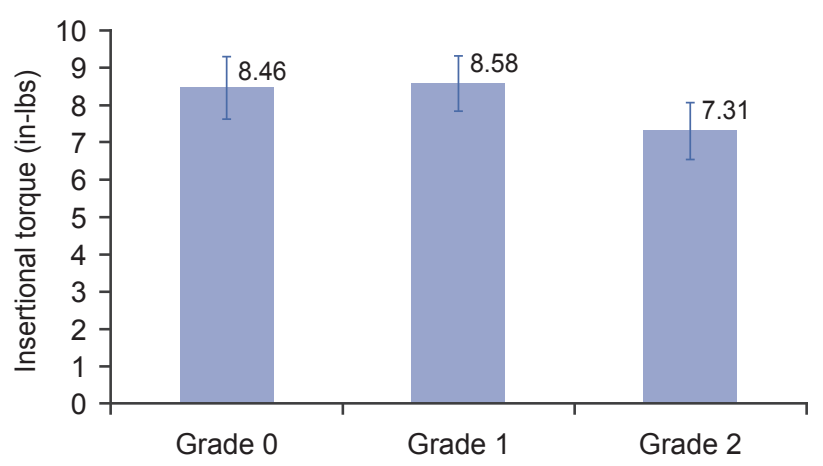

Fig. 5. The mean $\pm S E M$ for maximum insertional torque classified by a semiquantitative method. The differences were not significant. SEM, standard error of mean. traditional pedicle screws, which are inserted from the pedicle to the vertebral body and mainly anchored in the cancellous bone.

According to Wakabayasi et al. [8], the traditional pedicle screw fixation is not recommended for osteoporotic vertebrae (grades 2 and 3 on their Jikei scale) because of the appearance of a clear zone, which means a limitation for the screws. Meanwhile, Santoni et al. [1] hypothesized that their new technique would work well for patients with poor trabecular bone quality.

Our study was designed to clarify the extent to which the utility of this new technique would be influenced by osteoporotic vertebrae and how widely it could be used. For this investigation, it was essential to grade the various degrees of vertebral degeneration. In addition, we used insertional torque to make an indirect evaluation of bone quality of posterior elements and the relation between this and vertebral degeneration.

Many studies [9-11] have reported that the insertional torque of pedicle screws was highly correlated with pullout strength. Meanwhile, other studies describe screw loosening primarily caused by cyclic caudocephalad toggling at the bone-screw interface [12]; insertional torque cannot predict screw loosening [13]. However, pullout strength and screw toggling cannot be evaluated in vivo; therefore, the evaluation of insertional torque is thought to predict a patient's postoperative clinical and radiological course. Insertional torque is caused by frictional resistance between the screw threads and bones as well as radial compression of the trabeculae $[9,14]$. It can also be influenced by multiple other factors, such as screw length, pitch, thread, and shaft design and diameter as well as

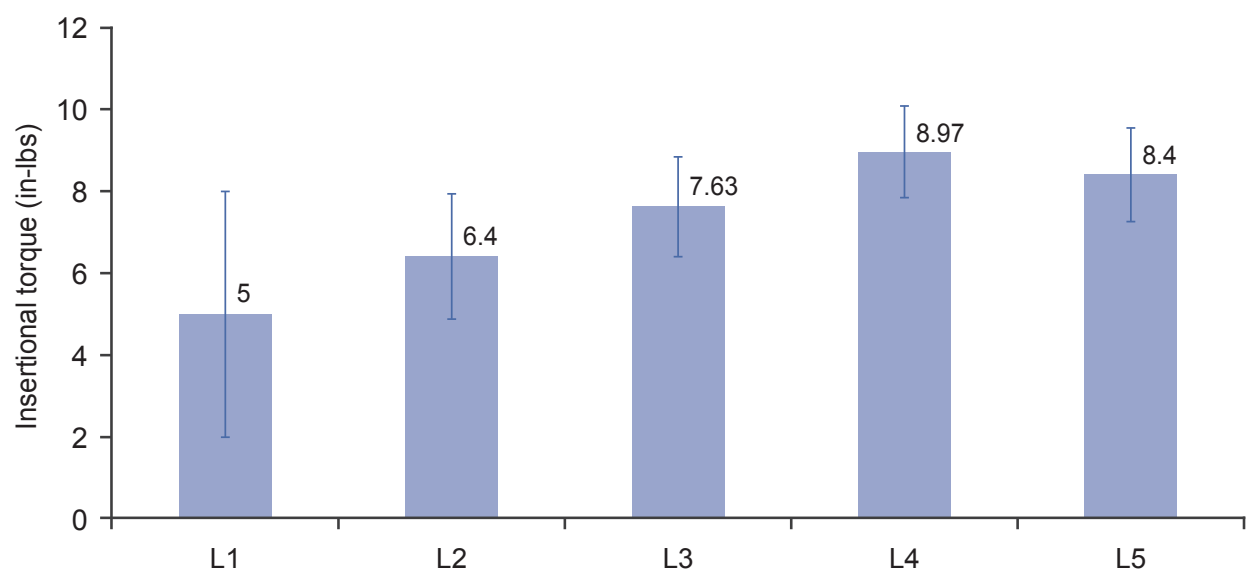

Fig. 6. The mean $\pm S E M$ for maximum insertional torque according to vertebral level. The differences were not significant. SEM, standard error of mean. 
bone mineral density (BMD) and surgical technique.

The in vivo insertional torque of CBT screws was first reported by Matsukawa et al. [2]. According to them, there was a significant difference between their mean maximum insertional torque $(2.49 \pm 0.99 \mathrm{Nm}=22.09 \pm 8.78 \mathrm{in}-\mathrm{lb})$ and that of traditional screws $(1.24 \pm 0.54 \mathrm{Nm}=11.00 \pm 4.79$ in-lb). They used SOLERA Spinal System screws with a diameter of $5.5 \mathrm{~mm}$, pitch of $2 \mathrm{~mm}$, and length ranging from 30 to $35 \mathrm{~mm}$. Their differential method of torque measurement and the larger size of screws with short pitches might explain their higher insertional torque than that observed in our study.

From an anatomical point of view, CBT screws are thought to be primarily stabilized at the cortex and trabecula of the neural arch, the middle or lateral side of the pedicle cortex, and the cortex of the vertebral edge. In spondylolysis, or a defect of the pars interarticularis, there is thus a partial lack of the cortex and trabecula in the neural arch. In our study, the insertional torque in cases with spondylolysis was significantly lower than that in those without spondylolysis. These observations highlight the importance of the cortex and trabecula of the pars interarticularis as an entry point for the CBT technique.

Some studies have reported a correlation between osteoporosis and the insertional torque of the traditional technique. Ozawa et al. [15] reported that there was a negative relation between the insertional torque of the lumbar traditional pedicle screw and the grade of osteoporosis, although they showed no significant relation between insertional torque and screw loosening or clinical outcome. Lee et al. [16] found that the insertional torque of the traditional pedicle screw had a positive correlation with BMD.

On the other hand, Matsukawa et al. [2] demonstrated that although positive linear correlations between maximum insertional torque and BMD were found in both techniques, the correlation coefficient of traditional screws was higher than that of the CBT screws.

Considering the relevance of these findings, the study performed by Giambini et al. [17] is very important. Through the study of 36 women aged $\geq 50$ years, they first showed that BMD of the neural arch trabecula was maintained regardless of the presence of a vertebral compression fracture. Therefore, compared with traditional screws, the insertional torque of CBT screws is thought to be less influenced by vertebral or femoral neck BMD.

In our study, instead of measuring vertebral BMD, we evaluated the relationship between the grade of vertebral degeneration and insertional torque. In addition, there was no statistical difference in the insertional torque, suggesting that BMD of the neural arch may be fairly maintained regardless of lumbar vertebral degeneration.

Our study demonstrated that late elderly women had significantly lower insertional torque than men $<75$ years. Because we could not clarify the reason for this finding, assessment of BMD in the neural arch according to sex and age may be needed, and it might be particularly important for this new technique.

Further studies will need to evaluate the relation between insertional torque and postoperative stability. However, our study highlights the importance of the pars interarticularis in the CBT technique because it does not appear to be affected by the lumber level or vertebral degeneration.

\section{Conclusions}

CBT is an efficient technique for the treatment of patients with osteoporosis and could therefore be used widely. However, in elderly women and patients with spondylolysis, careful consideration is advisable if potential complications are to be avoided.

\section{Conflict of Interest}

No potential conflict of interest relevant to this article was reported.

\section{References}

1. Santoni BG, Hynes RA, McGilvray KC, et al. Cortical bone trajectory for lumbar pedicle screws. Spine J 2009;9:366-73.

2. Matsukawa K, Yato Y, Kato T, Imabayashi H, Asazuma T, Nemoto K. In vivo analysis of insertional torque during pedicle screwing using cortical bone trajectory technique. Spine 2014;39:E240-5.

3. Iwatsuki K, Yoshimine T, Ohnishi Y, Ninomiya K, Ohkawa T. Isthmus-guided cortical bone trajectory for pedicle screw insertion. Orthop Surg 2014;6:2448.

4. Genant HK, Wu CY, van Kuijk C, Nevitt MC. Vertebral fracture assessment using a semiquantitative technique. J Bone Miner Res 1993;8:1137-48. 
5. Wu C, van Kuijk C, Li J, et al. Comparison of digitized images with original radiography for semiquantitative assessment of osteoporotic fractures. Osteoporos Int 2000;11:25-30.

6. Wu CY, Li J, Jergas M, Genant HK. Comparison of semiquantitative and quantitative techniques for the assessment of prevalent and incident vertebral fractures. Osteoporos Int 1995;5:354-70.

7. Ross PD, Fujiwara S, Huang C, et al. Vertebral fracture prevalence in women in Hiroshima compared to Caucasians or Japanese in the US. Int J Epidemiol 1995;24:1171-7.

8. Wakabayasi K, Matsuzaki H, Tokuhashi Y, et al. Indication and limitations of pedicle screw fixation systems for lumbar spinal stenosis: especially for osteoporotic spine. Kotsu Kansetsu Jintai 1998;11:3543.

9. Daftari TK, Horton WC, Hutton WC. Correlations between screw hole preparation, torque of insertion, and pullout strength for spinal screws. J Spinal Disord 1994;7:139-45.

10. Myers BS, Belmont PJ Jr, Richardson WJ, Yu JR, Harper KD, Nightingale RW. The role of imaging and in situ biomechanical testing in assessing pedicle screw pull-out strength. Spine (Phila Pa 1976) 1996;21:1962-8.

11. Zdeblick TA, Kunz DN, Cooke ME, McCabe R.
Pedicle screw pullout strength. Correlation with insertional torque. Spine (Phila Pa 1976) 1993;18:16736.

12. Law M, Tencer AF, Anderson PA. Caudo-cephalad loading of pedicle screws: mechanisms of loosening and methods of augmentation. Spine (Phila $\mathrm{Pa} 1976$ ) 1993;18:2438-43.

13. Okuyama K, Abe E, Suzuki T, Tamura Y, Chiba M, Sato K. Can insertional torque predict screw loosening and related failures? An in vivo study of pedicle screw fixation augmenting posterior lumbar interbody fusion. Spine (Phila Pa 1976) 2000;25:858-64.

14. Inceoglu S, Ferrara L, McLain RF. Pedicle screw fixation strength: pullout versus insertional torque. Spine J 2004;4:513-8.

15. Ozawa T, Takahashi K, Yamagata M, et al. Insertional torque of the lumbar pedicle screw during surgery. J Orthop Sci 2005;10:133-6.

16. Lee JH, Lee JH, Park JW, Shin YH. The insertional torque of a pedicle screw has a positive correlation with bone mineral density in posterior lumbar pedicle screw fixation. J Bone Joint Surg Br 2012;94:93-7.

17. Giambini H, Salman Roghani R, Thoreson AR, Melton LJ 3rd, An KN, Gay RE. Lumbar trabecular bone mineral density distribution in patients with and without vertebral fractures: a case-control study. Eur Spine J 2014;23:1346-53. 